\title{
EDITORIAL \\ Is preoperative bone health assessment and optimization in spine surgery a good idea?
}

\author{
Timothy F. Witham, MD, Ethan Cottrill, MS, and Zach Pennington, BS \\ Department of Neurosurgery and Orthopaedic Surgery, Johns Hopkins University School of Medicine, Baltimore, Maryland
}

A DEQUATE bone strength is critical in the setting of instrumented spinal fusion and deformity correction. Poor bone mineral density or bone quality has been correlated with decreased screw pullout strength, ${ }^{1}$ increased risk of vertebral compression fractures, ${ }^{2,3}$ and increased risk of instrumentation failure. ${ }^{4,5}$

With the increase in rates of fusion procedures ${ }^{6}$ and the aging US population, assessing preoperative bone strength or bone quality is of paramount importance. However, in individuals found to have poor bone quality, an assessment alone provides little benefit unless followed by means to optimize bone strength and thereby patient outcomes. At a minimum, all neurosurgeons and orthopedic surgeons performing instrumented spinal fusion procedures should have an awareness of bone health and the impact that poor bone quality can have on outcomes following spinal fusion. To this end, Anderson et al., in their paper "Preoperative bone health assessment and optimization in spine surgery," have taken steps toward improving patient outcomes related to bone health for spinal fusion procedures. ${ }^{7}$

The authors have set up a tertiary referral bone health optimization (BHO) clinic. They evaluated 104 patients who were being considered for thoracolumbar spinal surgery and were referred to the clinic over 1.5 years. Using National Osteoporosis Foundation (NOF) criteria, a very high percentage $(81.6 \%)$ of patients who had undergone dual-energy x-ray absorptiometry (DXA) were shown to have osteoporosis and were deemed candidates for preoperative BHO. Because these patients were referred to the $\mathrm{BHO}$ clinic at the request of the surgeon for suspected compromised bone health, this study does not describe the overall prevalence of osteoporosis in patients being considered for spinal fusion. Nonetheless, their data certainly suggest a high prevalence of poor bone quality in this population of patients. The methodology and logistics of setting up such a BHO clinic serve as a very useful model that could be easily implemented at other tertiary referral centers. At the same time, their article offers groundwork for practitioners who do not have the luxury of being at a tertiary care center, as it highlights some basic steps to begin an assessment of bone health for preoperative fusion patients.

We were surprised to see that $81 \%$ of patients had normal preoperative 25 -hydroxy vitamin $\mathrm{D}[25(\mathrm{OH}) \mathrm{D}]$ levels. In our experience, the percentage of patients with preoperative vitamin $\mathrm{D}$ deficiency is much greater. This relatively high percentage of patients with normal $25(\mathrm{OH}) \mathrm{D}$ levels in this study is likely reflective of the referring providers, who were spine surgeons, who likely understood the importance of calcium and vitamin D supplementation for bone health. Additionally, supplementation was also advised by the $\mathrm{BHO}$ clinic several weeks prior to testing.

While most would agree that standard methods for assessment of bone quality underestimate the true bone strength and hence the risk of instrumentation-related complications in spinal surgery, this paper highlights these points. By WHO guidelines, $32 \%$ of patients in this study were deemed to have osteoporosis. However, by NOF criteria, $81.6 \%$ were shown to have osteoporosis. A similarly high percentage $(72 \%)$ of patients was subsequently prescribed either antiresorptive agents or anabolic therapy. In fact, 44 of 104 patients (42\%) were treated with anabolic agents (abaloparatide or teriparatide). This is particularly important because of literature suggesting an improvement in spinal surgery outcomes with preoperative anabolic therapy. ${ }^{8,9}$

Is preoperative bone health assessment and optimization in spine surgery a good idea? The short answer is yes. The authors recognize that there is work left to be done in terms of demonstrating that preoperative BHO influences outcomes in our spinal fusion patients. It is the hope that the authors could evaluate the outcomes of surgery in this cohort of patients in a future report. Prospective observational studies would also be ideal for evaluating the 
efficacy of optimization interventions. Additional questions remain with regard to assessing true bone quality and strength as they relate to forces placed on the spine with spinal instrumentation. Perhaps quantitative CT data and even MRI data accumulated on a routine basis preoperatively will turn out to be a better correlate to bone strength at the surgical site. Future studies should also address the utility and indications for antiresorptive agents versus anabolic agents. Questions regarding timing will also be critical. What is the duration in advance of surgery that pharmacological agents should be administered? For how long after surgery should these agents be continued to maximize fusion results and bone healing? Lastly, can a BHO clinic determine in which patients spinal surgery might be prohibitive due to the unacceptably high risk of complications related to poor bone health?

We commend the authors for advancing this field of study.

https://thejns.org/doi/abs/10.3171/2020.5.FOCUS20414

\section{References}

1. Reitman CA, Nguyen L, Fogel GR. Biomechanical evaluation of relationship of screw pullout strength, insertional torque, and bone mineral density in the cervical spine. $J$ Spinal Dis Tech. 2004;17(4):306-311.

2. Lee BG, Choi J-H, Kim D-Y, et al. Risk factors for newly developed osteoporotic vertebral compression fractures following treatment for osteoporotic vertebral compression fractures. Spine J. 2019;19(2):301-305.

3. Ehresman J, Schilling A, Pennington Z, et al. A novel MRIbased score assessing trabecular bone quality to predict vertebral compression fractures in patients with spinal metastasis. J Neurosurg Spine. 2020;32(4):499-506.
4. DeWald CJ, Stanley T. Instrumentation-related complications of multilevel fusions for adult spinal deformity patients over age 65: surgical considerations and treatment options in patients with poor bone quality. Spine (Phila Pa 1976). 2006;31(19 suppl):S144-S151.

5. Bjerke BT, Zarrabian M, Aleem IS, et al. Incidence of osteoporosis-related complications following posterior lumbar fusion. Global Spine J. 2018;8(6):563-569.

6. Martin BI, Mirza SK, Spina N, Spiker WR, et al. Trends in lumbar fusion procedure rates and associated hospital costs for degenerative spinal diseases in the United States, 2004 to 2015. Spine. 2019;44(5):369-376.

7. Anderson PA, Kadri A, Hare KJ, Binkley N. Preoperative bone health assessment and optimization in spine surgery. Neurosurg Focus. 2020;49(2):E2.

8. Kim JW, Park SW, Kim YB, Ko MJ. The effect of postoperative use of teriparatide reducing screw loosening in osteoporotic patients. J Korean Neurosurg Soc. 2018;61(4):494-502.

9. Buerba RA, Sharma A, Ziino C, et al. Bisphosphonate and teriparatide use in thoracolumbar spinal fusion: a systematic review and meta-analysis of comparative studies. Spine. 2018;43(17):E1014-E1023.

\section{Disclosures}

Dr. Witham: advisory board member for, consultant for, and investor in Augmedics; grant support from the Gordon and Marilyn Macklin Foundation; and investor in Additive Orthopaedics, LLC. Mr. Cottrill: F30 grant from National Institute on Aging.

\section{Correspondence}

Timothy F.Witham: twitham2@jhmi.edu.

INCLUDE WHEN CITING

DOI: 10.3171/2020.5.FOCUS20414. 